\title{
THE EQUATION OF STATE
}

\section{WERNER DÄPPEN}

Department of Physics and Astronomy, University of Southern California, Los Angeles, CA 90089-1342, U.S.A. and

Institut für Astronomie, Türkenschanzstr. 17, 1180 Vienna, Austria

\begin{abstract}
There are two basic approaches to the equation of state for stellar envelopes and interiors. The traditional method chooses the so-called "chemical picture", in which the notion of atoms is maintained despite the plasma environment. A mixture of atoms, molecules, ions, electrons and nuclei is considered, and the occurring ionization and dissociation reactions (thus the name chemical picture) are treated according to the entropy-maximum (or free-energy-minimum) principle. The alternative method is based on the so-called "physical picture", where only fundamental particles (electrons, nuclei) explicitly enter. Through the means of activity expansions, the problems of plasma physics and statistical mechanics are treated simultaneously and on the same footing. For helio- and asteroseismology, an accurate and precise equation of state is essential. Progress towards a better equation of state can be made in several ways: purely theoretical efforts, checks with experiments, including astrophysical data, and comparisons between different theoretical formalisms. Comparisons are useful to assess the domain of temperature and density where the theoretical complications matter, and to determine the diagnostic potential of astrophysical observables for equation of state issues.
\end{abstract}

\section{INTRODUCTION}

The three basic material properties required in stellar models are the equation of state, opacity, and the nuclear-energy generation rate. Opacity is discussed by Seaton (these proceedings). A recent review on the equation of state and opacity is Däppen, Keady \& Rogers (1991). For a useful introduction to the equation of state see the book by Eliezer, Ghatak \& Hora (1986). The term equation of state has a narrower and a larger meaning. In the restricted sense, the (thermal) equation of state relates pressure to density and temperature. (The caloric equation of state expresses internal energy as a function of density and temperature). Thermal and caloric equations of state are derived from a single thermodynamic potential, such as e.g. the free energy. In a slightly broader sense, the term equation of state often means the associated thermodynamic potential. The thermodynamic quantities must be consistent with each other, that is, their appropriate Maxwell relations have to be satisfied. Such formal consistency is always achieved if the equation of state and the thermodynamic quantities stem 
from the same thermodynamic potential. In trivial models (e.g. in a plasma assumed to be fully ionized everywhere) it is possible to write down a consistent equation of state and thermodynamic quantities independently. However, in more realistic cases, modelling a thermodynamic potential is the only practical way to obtain a consistent equation of state and thermodynamic quantities.

Improvements in the equation of state beyond the model of a mixture of ideal gases are difficult. This has both conceptual and technical reasons. As a fundamental conceptual reason I mention the fact that in a plasma environment already the idea of isolated atoms (and compound ions) has to be abandoned. A technical reason is the difficulty encountered when specific non-ideal effect are modelled. The three principal non-ideal effects are related to: (i) the internal partition functions of bound systems, (ii) pressure ionization, and (iii) collective interactions of the charged particles. The internal partition functions contain the difficult problem of excited states, where and how they are to be cut off. They are an important element in determining the ionization balances. Pressure ionization has to be provided by non-ideal interaction terms, because ideal gases would unphysically recombine in the central regions of stars.

The plasma of the interiors of "normal" stars is only weakly non-ideal. One would therefore think that finding a good equation of state is not too difficult. Indeed, simple models of the equation of state have been highly successful in many calculations of stellar structure and evolution. However, the inadequacy of simple equations of state has been clearly demonstrated by helioseismology. The observed solar oscillation modes are standing acoustic waves; hence the quantity most obviously probed is local sound speed. Since the oscillations are largely adiabatic (except very near the surface), the frequencies are determined predominantly by the local adiabatic sound speed, which is a thermodynamic quantity. In addition, the frequencies depend on the density distribution in the Sun. A good knowledge of the equation of state is therefore very important for any interpretation of solar observational data. However, the remarkable accuracy and wealth of these data provides, in addition, powerful constraints on the equation of state (for a review see Christensen-Dalsgaard \& Däppen, 1992).

The smaller amount of expected stellar data will make a seismological determination of the equation of state more difficult. An accurate and precise equation of state is therefore a must. Obviously, all asteroseismic inferences will be subject to the uncertainty in the equation of state. However, not all applications require the same degree of accuracy. For instance, the calibration of solar evolutionary models to present-age radius and luminosity often reduces the influence on uncertainties in the equation of state. So, for instance, any uncertainty in the pressure in the solar centre is easily compensated with a slight change in the helium abundance. Many aspects of such a recalibrated model remain virtually unchanged and thus independent of the equation of state. However, if the same calibration is to yield the helium abundance, the result is affected by the whole uncertainty in the equation of state. As another example, the alternative helioseismic determination of the helium abundance in the solar convection zone (for recent progress see Kosovichev et al., 1992) does not profit from favourable cancellations. Since the key idea of this helium abundance determination is based on the signature of the second ionization zone of helium on the adiabatic exponent $\Gamma_{1}$, and thus on sound speed, an absolutely accurate equation of state is essential. Helioseismology has, in principle, the potential to 
localize thermodynamical properties everywhere in the Sun. Therefore, Gough (1984a) suggested to use the power of helioseismology to determine at the same time the equation of state and the helium abundance.

In the following, I will discuss the statistical-mechanical foundations of the equation of state needed for the interiors of "normal" stars. I will then discuss the requirements of helio- and asteroseismology and present two representative formalisms of current interest. I will use detailed comparisons between these two formalisms to assess the present uncertainty in the equation of state and to localize those regions in temperature and density where progress is most urgent. Comparisons will also be helpful to test the influence of controversial features in the equation of state on observational quantities.

\section{STATISTICAL MECHANICAL FOUNDATION OF THE EQUATION}

\section{QF STATE}

The typical task of quantum statistical mechanics consists in the calculation of the (total) partition function (see, e.g., Huang, 1963, Reichl, 1980)

$$
Z(T, V, \mathrm{~N})=\operatorname{Tr}\left(\mathrm{e}^{-H / k T}\right) .
$$

Here, $H$ is the Hamiltonian operator of the quantum system with $N_{i}$ particles of species $i, i=1, \ldots, m$ [i.e., $\mathbf{N}=\left(N_{1}, N_{2}, \ldots, N_{m}\right)$ ] confined to a box of volume $V$, and $k$ is the Boltzmann constant. (Note that this is the canonical point of view; the alternative, equivalent grand-canonical approach is another possibility, which is discussed in a subsequent section). The partition function directly leads to the thermodynamical potential of the free energy

$$
F(T, V, \mathbf{N})=-k T \ln Z(T, V, \mathbf{N}),
$$

from which all thermodynamic quantities can be derived. Given the large number of interacting particles, it is evident that various approximations are necessary before Eq. (1) can be evaluated. One begins with treating the motion of the heavy particles (nuclei, atoms, ions) according to classical mechanics, which is certainly appropriate as long one does not approach the conditions of the interior of neutron stars. Thus, only electrons have to be described by quantum mechanics.

Such a separation of effects leads to a factorization in Eq. (1), which is translated into a sum in Eq. (2). The free energy thus becomes modular, which is a very useful property of models based on the canonical partition function. The classical contribution in Eq. (1) is obtained from an integration over the Hamiltonian coordinates $p_{i}$ and $q_{i}(i=1, \ldots, 3 N)$. Neglecting velocity-dependent interactions between the particles, the classical integrations over $p_{i}$ and $q_{i}$ factorize. The result is a product of the Maxwell-Boltzmann distribution for the momenta of heavy particles with the so-called configuration integral

$$
Q(T, V, \mathrm{~N})=\int d p_{1} \cdots d p_{3 N} d q_{1} \cdots d q_{3 N} \mathrm{e}^{-V^{i n t} / k T},
$$

where $V^{i n t}=V^{i n t}\left(q_{1}, \ldots, q_{3 N}\right)$ is the interaction potential of the heavy particles. Equation (3) is at the base of the (negative) Coulomb-pressure correction 
for the charged particles and of excluded-volume effects of neutral particles (such as hard-sphere and Van-der-Waals type corrections). Excluded volume effects become very important in relatively cold plasmas of low-mass stars or planets (see Saumon \& Chabrier, 1991,1992; Chabrier \& Saumon, these proceedings). In the Coulomb case, charge neutrality requires the electrons to be included, though for consistency with the assumptions of Eq. (3) they have to be treated classically (but quantum correction factors can be included). An approximate evaluation of the Coulomb pressure correction is made in the Debye-Hückel theory, where it is assumed that each charge is surrounded by a cloud of opposite charge, which screens the Coulomb interaction outside a sphere with the so-called Debye radius. In many astrophysical equations of state the specific form of the Debye-Hückel Coulomb free energy used is that adopted by Graboske, Harwood \& Rogers (1969). There, two additional effects are taken into account. First, with increasing partial degeneracy of the electrons, their influence in Eq. (3) is gradually reduced (by a factor involving Fermi integrals), and second, the configurational integral $Q$ appears with a multiplicative correction factor $\tau$ that serves as a rough approximation for the non-vanishing size of the charged particles (see also Chabrier, 1990; Ebeling et al., 1991). The Debye-Hückel approximation is basically valid as long as there are still relatively many particles in the so-called Debye sphere (with the Debye length as its radius).

Once the heavy particles are separated out, electrons remain, and they should be, of course, treated according to quantum mechanics. Here, there is a bifurcation into two distinct classes of approach, the "chemical picture" and the "physical picture" (Krasnikov, 1977). While in the more conventional chemical picture bound configurations (atoms, ions and molecules) are introduced and treated as new and independent species, only fundamental particles (electrons and nuclei) appear in the physical picture. In the chemical picture, reactions between the various species occur, and thus the thermodynamical equilibrium must be sought among the stoichiometrically allowed set of concentration variables by means of a maximum entropy (or minimum free-energy) principle. In contrast, the physical picture has the aesthetic advantage that there is no need for a minimax principle; the question of bound states is dealt with implicitly through the Hamiltonian describing the interaction between the fundamental particles. The different physical assumptions of the two pictures have been the source of considerable difficulties in recent comparisons and interpretations (see, e.g., Rouse, 1983; Ebeling et al., 1985; Däppen, Anderson \& Mihalas, 1987).

There is an intuitive simplicity in the chemical picture: we usually take the existence of atoms in plasmas for granted, at least at densities that are not too high. However, this simplicity has to be paid for by additional minimization procedures in the multidimensional space of abundances of each species, restricted by the appropriate stoichiometrical relations and by mass and charge conservation. The physical idea behind this minimization is simple: the "internal" degrees of freedom, such as ionization degrees, are not adjustable by the experimenter; one can only control "external" parameters, like temperature, density, and mass fractions of each chemical element. The thermodynamic equilibrium is then determined as the one configuration, compared with those having different internal parameters, that minimizes the free energy, or equivalently, maximizes entropy. Once this minimum is found, the model free energy delivers all thermodynamic quantities in a straightforward way by differentiation. 
It should be clear that the advantage of the chemical picture lies in the possibility to model complicated plasmas, and to obtain numerically smooth and consistent thermodynamical quantities (see below). Nevertheless, the heuristic method of the separation of the atomic-physics problem from that of statistical mechanics is not satisfactory, and attempts have been made to avoid the concept of a perturbed atom in a plasma altogether. Thus in the physical picture only fundamental particles (electrons and nuclei) enter. This has the advantage that the constraints appearing in the chemical picture (i.e., mass and charge conservation) are automatically satisfied. In the absence of constraints, it becomes possible to use the powerful apparatus of the grand-canonical partition function. In the chemical picture, however, this road is barred, because the aforementioned constraints are expressed in numbers of particles and not in terms of the chemical potentials, the independent variables of the grand-canonical partition function. In the physical picture, one can build a theory of partially ionized plasmas similar to well-know cluster expansions for real gases (Rogers, 1981; for an introduction into cluster expansions see Huang, 1963).

\section{STELLAR DEMANDS ON AN EQUATION OF STATE}

It is important to realize that a stellar equation of state has to be formally precise and consistent, even before the question of the accuracy of the physical description is asked. It has to satisfy four conditions: i) a large domain of applicability (in $\rho, T$ ), ii) a high precision of its numerical realization, iii) consistency between the thermodynamic quantities, and $i v)$ the possibility to take into account relatively complex mixtures with at least several of the more abundant chemical elements.

More specifically, the first condition demands that the formalism can be used from the stellar surface (the photosphere), where $T$ is typically a few $10^{3} \mathrm{~K}$ and $\rho$ some $10^{-7} \mathrm{~g} / \mathrm{cm}^{3}$, to the center of a star where $T$ is, again typically, about $10^{7} \mathrm{~K}$ and $\rho$ some $10^{2} \mathrm{~g} / \mathrm{cm}^{3}$. The second condition demands that a given formalism can be cast in an algorithm that converges without ambiguity and with sufficient precision, so that all required thermodynamic derivatives (such as adiabatic gradients) can be computed. Note, that for this only formal precision is required: reality of the physical description is a different issue. The third condition, consistency, states that all thermodynamic quantities stem from a single thermodynamic potential. This condition is often violated in two- or morezone formalisms, which contains a different physical theory in different parts of a star. An example is the ad hoc imposition of full ionization in the central region, in order to mimic a pressure-ionization device, in combination with a conventional Saha equation in the envelope of the star. Such a formalism leads to a discontinuous thermodynamic potential and a violation of thermodynamic identities. Thermodynamic identities are, however, often used in calculations of stellar structure and oscillations. As an example of the use of thermodynamical identities, I mention the transformation of the density fluctuation to the pressure fluctuation in linear adiabatic pulsation calculations. The connection between density and pressure changes is given by the adiabatic gradient $\Gamma_{1}$, and it is therefore imperative that this gradient is consistent with the equation of state and other thermodynamic variables used in the model. This example illustrates 
the necessity of formal consistency. Finally, the third and last condition, i.e. the possibility to describe rather realistic chemical compositions, is less important for the equation of state itself. However, for opacity, heavy elements are important, and a good equation of state plays an important role in any opacity calculation.

It is in view of these specific requirements that astrophysicists dare to develop their own formalisms, which are often built around time-honoured intuitive ideas, and therefore lack a rigorous foundation. One of these "home-grown" astrophysical equations of state has been developed as part of the international "Opacity Project" (OP, see Seaton, 1987,1992, and these proceedings) by Mihalas, Hummer and Däppen (Hummer \& Mihalas, 1988; Mihalas, Däppen \& Hummer, 1988; Däppen et al., 1988; hereinafter MHD). The MHD equation of state is written in the chemical picture. Plasma interactions are treated with modifications of atomic states, i.e. the quantum mechanical problem is solved before statistical mechanics is applied.

Of course, many more-or-less rigorous formalisms for strongly-coupled plasmas have been developed, and extensive numerical simulations have been made (see, e.g., the book by Ebeling et al., 1991, and references therein). However, since their motivation is different, they mostly aim at understanding qualitative phenomena, and they are normally not suited for the purposes of stellar models. That is, their results are not sufficiently precise, nor do they allow a description of realistic astrophysical mixtures. They would therefore not satisfy the four conditions mentioned above. A first attempt to develop a stellar equation of state with a better statistical mechanical foundation was made as part of an opacity project at Livermore (OPAL) (Rogers, 1986; Iglesias, Rogers \& Wilson, 1987; Iglesias \& Rogers, 1991; hereinafter Livermore equation of state).

TWO EXAMPLES OF EQUATIONS OF STATE: CHEMICAL AND PHYSICAL PICTURE

The MHD equation of state (chemical picture)

In the chemical picture, perturbed atoms must be introduced on a more-or-less ad-hoc basis to avoid the familiar divergence of internal partition functions (see e.g. Ebeling, Kraeft \& Kremp, 1976). In other words, the approximation of unperturbed atoms precludes the application of standard statistical mechanics, i.e. the attribution of a Boltzmann-factor to each atomic state. The conventional remedy of the chemical picture against this is a modification of the atomic states, e.g. by cutting off the highly excited states in function of density and temperature of the plasma. Such cut-offs, however, have in general dire consequences due to the discrete nature of the atomic spectrum, i.e. jumps in the number of excited states (and thus in the partition functions and in the free energy) despite smoothly varying external parameters (temperature and density).

The MHD equation of state avoids these discontinuities (in the free energy) by introducing "soft" cut-offs in the form of occupational probabilities. These occupation probabilities have the same function as the "hard" cut-offs mentioned above. The occupational probabilities of a state simulate a result from quantum mechanics, denoting the fraction of atoms where the state can exist. Only then, these "available" states are populated according to statistical mechanics. It is clear that such an approach is largely intuitive. However, its advantage 
is that complicated plasmas can be modelled, with detailed internal partition functions of a large number of atomic, ionic, and molecular species. Also, full thermodynamic consistency is assured by analytical expressions of the free energy and its first- and second-order derivatives. This not only allows an efficient Newton-Raphson minimization, but, in addition, the ensuing thermodynamic quantities are of analytical precision and can therefore be differentiated once more, this time numerically. Reliable third-order thermodynamic quantities are thus calculated.

In the MHD occupation probabilities, perturbations by charged and neutral particles are taken into account. Correlations between the two effects are neglected (for lack of knowing how to describe them); thus the occupation probabilities due to charged and neutral perturbers are simply multiplied. The re-

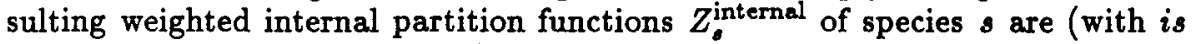
labelling the state $i$ of species $s$ )

$$
Z^{\text {internal }}=\sum_{i} w_{i s} g_{i s} \exp \left[-\frac{E_{i s}-E_{1 s}}{k T}\right]
$$

The coefficients $w_{i,}$ take into account charged and neutral surrounding particles. In physical terms, $w_{i s}$ gives the fraction of all particles of species $s$ that can exist in state $i$ with an electron bound to the atom or ion, and $1-w_{i}$ gives the fraction of those that are so heavily perturbed by nearby neighbours that the state is effectively destroyed. Perturbations by neutral particles are based on an excluded volume treatment and perturbations by charges are calculated from a fit to a quantum-mechanical Stark-ionization theory. Hummer and Mihalas's (1988) choice has been

$$
\ln w_{i s}=-\left(\frac{4 \pi}{3 V}\right)\left\{\sum_{\nu} N_{\nu}\left(r_{i s}+r_{1 \nu}\right)^{3}+16\left[\frac{\left(Z_{s}+1\right) e^{2}}{\chi_{i,} k_{i s}^{1 / 2}}\right]^{3} \sum_{\alpha \neq e} N_{\alpha} Z_{\alpha}^{3 / 2}\right\}
$$

Here, the index $\nu$ runs over neutral particles, the index $\alpha$ runs over charged ions (except electrons), $r_{i,}$ is the radius assigned to a particle in state $i$ of species $s, \chi_{i s}$ is the (positive) binding energy of such a particle, $k_{i s}$ is a quantummechanical correction, and $Z_{s}$ is the net charge of a particle of species $s$. Note that $\ln w_{i s} \propto-n^{6}$ for large principal quantum numbers $n$ (of state $i$ ), and hence

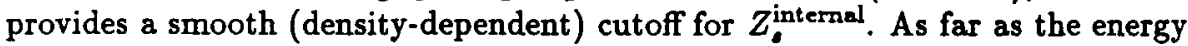
levels are concerned, in the MHD equation of state no shifts due to plasma effects are assumed. This choice is based on experimental and theoretical arguments (see Hummer \& Mihalas, 1988). Finally, the MHD equation of state also includes a Debye-Hückel term for the Coulomb-pressure correction, partially degenerate electrons, and radiation pressure.

The Livermore equation of state (physical picture)

There is an impressive body of literature on the physical picture. Important sources of information with many references are the books by Ebeling, Kraeft \& Kremp (1976), Kraeft et al. (1986), Ebeling et al. (1991). However, the majority of work on the physical picture was not dedicated to the problem of obtaining a high precision equation of state for stellar interiors. Such an attempt 
was made for the first time by a group at livermore as part of an opacity project (Rogers, 1986; Iglesias, Rogers \& Wilson, 1987; Iglesias \& Rogers, 1991,1992).

It is clear from the preceding subsection that the advantage of the chemical picture lies in the possibility to model complicated plasmas, and to obtain numerically smooth and consistent thermodynamical quantities. Nevertheless, the heuristic method of the separation of the atomic-physics problem from that of statistical mechanics is not satisfactory, and attempts have been made to avoid the concept of a perturbed atom in a plasma altogether. This has suggested an alternative description, the physical picture. In such an approach one expects that no assumptions about energy-level shifts or the convergence of internal partition functions have to be made. On the contrary, properties of energy levels and the partition functions should come out from the formalism.

To explain the advantages of this approach for partially ionized plasmas, it is instructive to discuss the activity expansion for gaseous hydrogen. The interactions in this case are all short ranged and pressure is determined from a self-consistent solution of the equations (Rogers, 1981)

$$
\begin{gathered}
\frac{p}{k T}=z+z^{2} b_{2}+z^{3} b_{3}+\ldots \\
\rho=\frac{z}{k T}\left(\frac{\partial p}{\partial z}\right)
\end{gathered}
$$

where $z=\lambda^{-3} \exp (\mu / k T)$ is the activity, $\lambda \equiv h / \sqrt{2 \pi m_{e} k T}$ is the thermal (de Broglie) wavelength of electrons, $\mu$ is the chemical potential and $T$ is the temperature. The $b_{n}$ are cluster coefficients such that $b_{2}$ includes all two particle states, $b_{3}$ includes all three particle states, etc.. More specifically, the second cluster coefficient for hydrogen includes the formation of $\mathrm{H}_{2}$ molecules as well as the scattering states of the $\mathrm{H}$ atoms. The states of the $\mathrm{H}-\mathrm{H}$ system are of different type according to the configurations of the electrons. Molecules are always in the singlet state, with total electron spin $S=0$, and the lowest symmetrical orbital wave function, the ${ }^{1} \Sigma_{g}$ bonding orbital. In this configuration, the interaction between the $\mathrm{H}$ atoms is described by the attractive ${ }^{1} \Sigma_{g}$ bonding potential. The scattering states of the $\mathrm{H}$ atoms are either singlet states, with sufficient kinetic energy of the $\mathrm{H}$ atoms so that they are in the continuum of the ${ }^{1} \Sigma_{g}$ bonding potential. Or, they belong to the triplet states (total electron spin $S=1$ ), for which even the lowest orbital (the ${ }^{3} \Sigma_{u}$ antisymmetric orbital) leads to a repulsive ${ }^{3} \Sigma_{u}$ antibonding potential, which, of course, has only scattering states of the $\mathrm{H}-\mathrm{H}$ system. Or, they belong to excited electronic states (singlet and triplet), which are never bound, either.

As a consequence, the second cluster coefficient for hydrogen includes the formation of $\mathrm{H}_{2}$ molecules, the scattering states in the ${ }^{1} \Sigma_{g}$ potential, in the ${ }^{3} \Sigma_{u}$ potential, and in the potentials of all excited electronic states. The third cluster coefficient includes $\mathrm{H}_{3}$ bound states, $\mathrm{H}-\mathrm{H}_{2}$ and $\mathrm{H}-\mathrm{H}-\mathrm{H}$ scattering states. Eq. (6) demonstrates that the equation of state for reacting gases can be obtained without an explicit knowledge of the occupation numbers of the internal states of the composite particles.

For low-density gases the bound-state contributions to the $b_{n}$ can be important at low temperature while the scattering contributions are too small to 
matter. Strict application of Eq. (6) would contain a large amount of unimportant information which is very hard to calculate. Consequently it is necessary to reorganize Eq. (6) such that the bound-state terms from each $b_{n}$ are treated as being of the same order as the ideal gas term, i.e., of order $z$. Terms of order $z$ in the physical picture are roughly equivalent to what in the chemical picture is called the Saha equation.

In the case of partially ionized plasmas very similar steps are required, except that now even Eq. (6) must involve at least two species (nuclei and electrons) to assure electrical neutrality. In addition, due to the long range of the Coulomb potential each of the $b_{n}$ is composed of a number of divergent terms, some of which are fictitious and some of which are real.

An example of real divergence is afforded by the classical ring diagrams occurring in each $b_{n}$ (Mayer, 1950). They are individually divergent, but the many-body correlations introduced by summing over the $b_{n}$ yield the DebyeHückel correction [Eq. (6)]. This type of divergence occurs even for an electron gas in a neutralizing background for which there are no bound states. Although the original equations involved only even powers in the activity as a result of many-body Coulomb correlations, the Debye-Hückel term appears in the power of $z^{3 / 2}$ in the activity (also in density).

An important example of a fictitious divergence is that associated with the atomic partition function. This divergence is fictitious in the sense that the bound-state part of $b_{2}$ is divergent but the scattering state part, which is omitted in the Saha approach, has a compensating divergence. Consequently the total $b_{2}$ does not contain a divergence of this type (Ebeling, Kraeft \& Kremp, 1976; Rogers, 1977). A major advantage of the physical picture is that it incorporates this compensation at the outset. A further advantage is that no assumptions about energy-level shifts have to be made (see the previous subsection); it follows from the formalism that there are none.

As a result, the Boltzmann sum appearing in the atomic (ionic) free energy is replaced with the so-called Planck-Larkin partition function (PLPF), given by (Ebeling, Kraeft \& Kremp, 1976; Kraeft et al., 1986)

$$
\text { PLPF }=\sum_{n l}(2 l+1)\left[\exp \left(-\frac{E_{n l}}{k T}\right)-1+\frac{E_{n l}}{k T}\right] .
$$

The PLPF is convergent without additional cut-off criteria as are required in the chemical picture. I stress, however, that despite its name the PLPF is not a partition function, but merely an auxiliary term in a virial coefficient (see, for example, Däppen et al., 1987).

\section{EQUATION OF STATE COMPARISONS}

So far there are no laboratory experiments that could distinguish between equations of state in the chemical and physical picture. Attempts to use constraints from a high-precision optical emission spectrum (e.g. Wiese, Paquette \& Kelleher, 1973) have failed, because line-broadening effects were overshadowing the subtle details of statistical mechanics. The current equations of state (chemical and physical picture) all succeed in modelling the experiment (Däppen, Anderson and Mihalas, 1987; Seaton, 1990; Iglesias \& Rogers, 1992). As another 

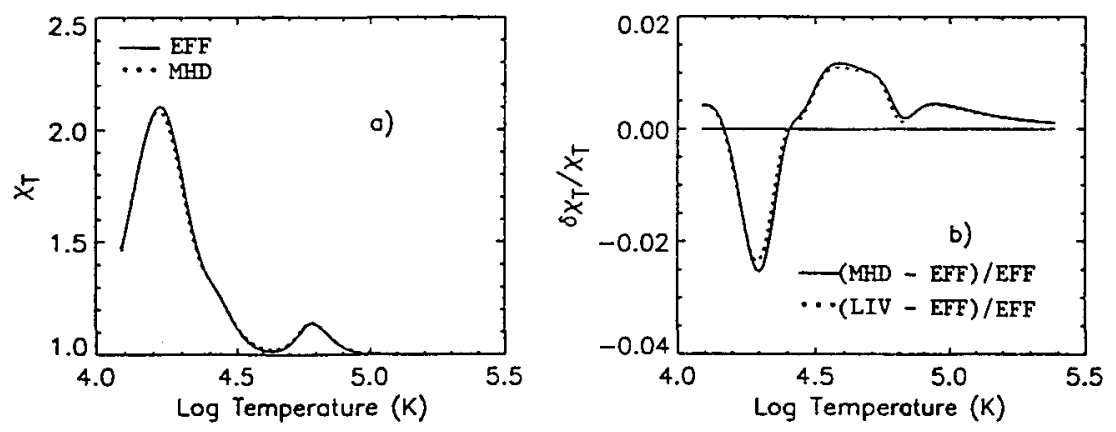

FIGURE I Comparison of $\chi_{T}=(\partial \ln p / \partial \ln T)_{\rho}$ for $\rho=10^{-6.5} \mathrm{~g} \mathrm{~cm}^{-3}$. Absolute quantities (a) and relative differences (with respect to EFF) (b) are shown. See text for more details.

type of observational diagnosis, solar oscillations promise to test the equation of state in the near future (see Christensen-Dalsgaard, Däppen \& Lebreton, 1988; Christensen-Dalsgaard, 1991; Christensen-Dalsgaard \& Däppen, 1992). Another way to learn about the merits of individual equations of states is with the help of theoretical comparisons. In addition, such comparisons also allow solar physicists to determine how uncertainties in the equation of state propagate into theoretically predicted oscillation frequencies. In this way, a "map" of the $T-\rho$ plane can be drawn, showing localized "interesting" regions, where non-ideal effects of one or another kind are important.

In the following, I discuss what has so far emerged from such comparisons. An early comparisons showed a striking agreement between the MHD and Livermore equation of state for conditions as found in the hydrogen-helium ionization zones of the Sun (Däppen, Lebreton \& Rogers, 1990; Däppen, 1990). For convenience, a representative result from this early comparison is shown in Figure 1, which compares the MHD and Livermore results with that of the simple Eggleton, Faulkner \& Flannery (1973; hereinafter EFF) formalism (which is essentially a consistent ground-state-only Saha equation of state including a - here irrelevant - arbitrary pressure-ionization device). The absolute curves of part $a$ of Figure 1 are merely able to show the difference between MHD (or Livermore) and the simple EFF results. To see the difference between the MHD and Livermore results, one needs the magnified part $b$, which shows the relative differences between MHD and EFF, and between Livermore and EFF values, respectively. This relative plot now not only allows one to see the difference between MHD and Livermore results, but also their striking similarity.

Later, it turned out that this agreement was nearly accidental. Its physical reason was found by varying the parameters of the MHD equation of state. It followed that on the chosen isochore, all thermodynamical quantities are mainly dominated by the Coulomb pressure correction (Däppen, 1990; ChristensenDalsgaard, 1991; Christensen-Dalsgaard \& Däppen, 1992). The Coulomb correction overshadows the effect of the excited states (which are of course treated differently in the MHD and Livermore approach). However, the Coulomb term acts principally indirectly, at least in the language of the chemical picture, be- 
cause it is not mainly the free-energy of the Debye-Hückel term itself, but rather the Coulomb-induced shift in the ionization equilibrium, which is responsible for the deviation from the unperturbed EFF result.

Of course, solar physicists were happy that two completely different formalisms delivered the same equation of state, but, by the same token, a first attempt to use the Sun as an equation-of-state test was also thwarted. This discovery suggested to upgrade the simple EFF equation of state with the help of the Coulomb interaction term. The resulting equation of state (called CEFF) has become a useful tool for solar physics (Christensen-Dalsgaard, 1991; ChristensenDalsgaard \& Däppen, 1992); at the same time, however, it became also clear that a helioseismic test of the important issue of chemical versus physical picture would be more difficult than first thought.

For reasons not yet fully understood it seems that in the chemical picture, the signature of internal partition functions, such as those employed in the MHD equation of state, is much less visible in the thermodynamic quantities than a naive estimation of the shift in the ionization equilibrium would predict. It is likely that there are accidental cancellations in the derivatives of the free energy. Notice that these cancellation would have nothing to do with those appearing in the physical picture, which lead to the Planck-Larkin partition function.

The cancellations of partition-function effects in the chemical picture seem to be greatest for the ionization zone of hydrogen and somewhat less for those of helium. A more recent comparison of MHD and Livermore values (Däppen, 1992) has examined selected cases of higher densities (where sizeable discrepancies appear) and a first case of a mixture involving a representative solar heavy element (oxygen). It appears that for the heavier elements, the internal partition functions finally lead to the intuitively expected consequences for the thermodynamic quantities. For convenience, Figure 2 shows the result of this comparison with oxygen for the quantity $\Gamma_{1}$. Density was chosen as $\rho=0.005 \mathrm{~g} \mathrm{~cm}^{-3}$, a value suggested by a helioseismic study of the solar helium abundance (Kosovichev et al., 1992). Here, not only do the large MHD partition functions cause shifts in the ionization balance but these shifts also significantly propagate into the thermodynamic quantities. The effect is large enough that it appears, despite the small relative number of the heavy elements in the mixture, to be within reach of helioseismology (Christensen-Dalsgaard \& Däppen, 1992). Incidentally, it is precisely this effect that was mainly responsible for the discrepancies found between the different sets of MHD tables (MHD1 - MHD5) discussed in Kosovichev et al. (1992). Those different MHD tables were computed for various purposes (some of them with limited computing resources), and they differed by their choice of heavy-element composition (see also Däppen et al., these proceedings).

To examine the MHD ionization fractions, a single case was examined $(T=$ $2.10 \times 10^{5} \mathrm{~K}, \rho=5.00 \times 10^{-3} \mathrm{~g} \mathrm{~cm}^{-3}$ ), once with the full MHD equation of state, once with a "stripped-down" version of MHD, which does not contain any excited states (but is otherwise identical). The resulting ionization fractions of $\mathrm{O}^{3+}, \mathrm{O}^{4+}, \mathrm{O}^{5+}$ were, respectively, $0.314,0.248,0.364$ for the stripped-down MHD (without excited states), and $0.304,0.476,0.182$ for the full MHD. (The result for the stripped-down very closely reflects the ground-state weights of the ions). Not unexpectedly in view of the Planck-Larkin partition function, the Livermore equation of state predicts ionization fractions close to those of the 

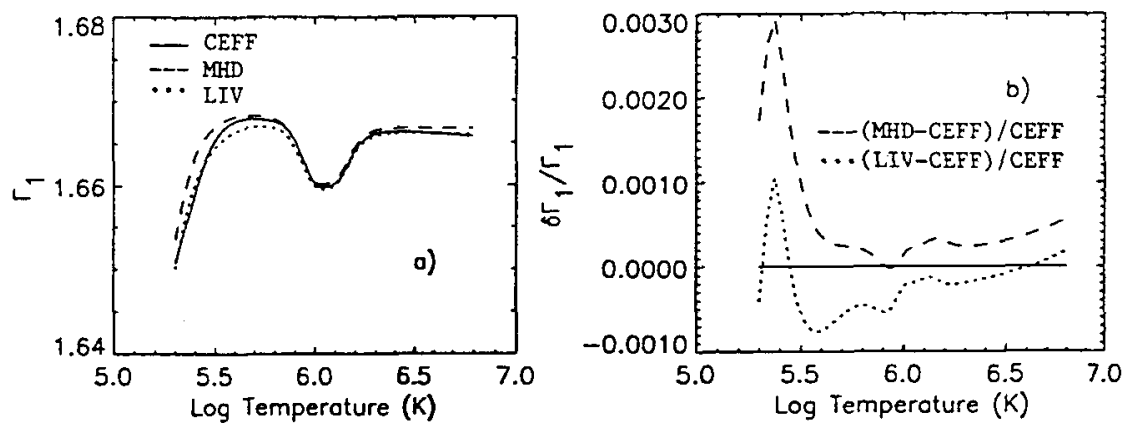

FIGURE II $\Gamma_{1}$ for $\rho=5.00 \times 10^{-3} \mathrm{~g} \mathrm{~cm}^{-3}$ and a representative solar mixture of $\mathrm{H}, \mathrm{He}$, and $\mathrm{O}$. Parts (a) and (b) as in Fig.1, but here with CEFF instead of EFF. See text for more details.

stripped-down MHD equation of state (Rogers, private communication).

This comparison for the first time establishes a clear case of disagreement between the MHD and Livermore results. Clearly, the origin of the discrepancy in the ionization degrees is due to the treatment of the excited states. Of course, only some 2 percent of the matter in the Sun consist of elements heavier that $\mathrm{H}$ and $\mathrm{He}$, and therefore the signature of the MHD-Livermore discrepancy (Figure 2) is small (of the order of $10^{-3}$ ). Nevertheless, as has been demonstrated by Christensen-Dalsgaard \& Däppen (1992), even the resulting tiny sound-speed differences are within reach of a helioseismic diagnosis.

\section{CONCLUSION}

Even weakly-coupled plasmas can pose tough problems if high accuracy is demanded. Solar oscillations are an example of a case where the present observational material is much better than the theoretical models. Stellar oscillations also promise interesting constraints. Their observations will not be as detailed as those of the Sun: however, a sample of more than one star will access a variety of different physical conditions and chemical compositions. A great many of astrophysical inferences will only be as good as the equation of state. The theoretical models used for the inferences will suffer from inconsistencies and bad numerical realizations of any formalism of the equation of state. These formal considerations are even above the question of an absolutely accurate representation of reality. At the moment, there is not much available to check the absolute accuracy of an equation of state. Solar and stellar seismology will undoubtedly play an important role. So far, theoretical comparisons between different formalisms are the only means to do this, but of course agreement does not mean correctness. Nevertheless, comparisons certainly give valuable indications, not to the least about the correct realization of the models. In addition, the comparisons are very important to localize the interesting regions in the $T$ - $\rho$ plane, where different formalisms lead to distinct thermodynamic quantities, which has observable consequences. For the Sun, such comparisons have already resulted 
in a better (and yet simple) equation of state (i.e. CEFF). There is hope that for stars, too, new data from ground-based and space projects will put constraints on the equation of state and shed light on controversial issues.

\section{ACKNOWLEDGMENTS}

I am grateful to Vladimir Baturin, Jørgen Christensen-Dalsgaard, Andreas Förster, Douglas Gough, and Forrest Rogers for stimulating discussions and helpful comments. Forrest Rogers is also thanked for providing the Livermore results displayed in the figures.

\section{REFERENCES}

Chabrier, G.: 1990, J. de Physique (France) 51, 1607

Christensen-Dalsgaard, J.: 1991, In Lecture Notes in Physics, Vol. 388: Challenges to Theories of the Structure of Moderate-mass Stars, eds Gough, D.O. \& Toomre, J., Springer, Heidelberg, p. $11-36$

Christensen-Dalsgaard, J. \& Däppen, W.: 1992, Astron. Astrophys. Review submitted

Christensen-Dalsgaard, J., Däppen, W. \& Lebreton, Y.: 1988, Nature 336, 634 $-638$

Däppen, W.: 1990, in Lecture Notes in Physics, Vol. 367: Progress of Seismology of the Sun and Stars, eds. Osaki, Y. \& Shibahashi, H., Springer, Berlin, p. $33-40$

Däppen, W.: 1992, in Astrophysical Opacities, eds. C. Mendoza \& C. Zeippen (Revista Mexicana de Astronomía y Astrofísica) $141-149$

Däppen, W., Anderson, L.S. \& Mihalas, D.: 1987, Astrophys. J. 319, $195-206$

Däppen, W., Keady, J. \& Rogers, F.: 1991, in Solar Interior and Atmosphere, eds Cox, A.N., Livingston, W.C. \& Matthews, M., Space Science Series, University of Arizona Press, Tucson, p. $112-139$

Däppen, W., Lebreton, Y. \& Rogers, F.: 1990, Solar Physics 128, 35 - 47

Däppen, W., Mihalas, D., Hummer, D.G. \& Mihalas, B.W.: 1988, Astrophys. J. 332, $261-270$

Ebeling, W., Kraeft, W.D. \& Kremp, D.: 1976, Theory of Bound States and Ionization Equilibrium in Plasmas and Solids, Akademie Verlag, Berlin, DDR

Ebeling, W., Kraeft, W.D., Kremp, D. \& Röpke, G.: 1985, Astrophys. J. 290 $24-27$

Ebeling, W., Förster, A., Fortov, V.E., Gryaznov, V.K. \& Polishchuk, A.Ya.: 1991, Thermodynamic Properties of Hot Dense Plasmas, Teubner, Stuttgart, Germany

Eggleton, P.P., Faulkner, J. \& Flannery, B.P.: 1973, Astron. Astrophys. 23, 325 $-330$ 
Eliezer, S., Ghatak, A. \& Hora, H.: 1986, An introduction to equations of state: theory and applications, Cambridge University Press

Gough, D.O.: 1984a, Mem. Soc. Astron. Ital. 55, $13-35$

Graboske, H.C., Harwood, D.J. \& Rogers, F.J.: 1969, Phys. Rev. A186, 210

Huang, K.: 1963, Statistical Mechanics, John Wiley, New York, Chapt. 14

Hummer, D.G. \& Mihalas, D.: 1988, Astrophys. J. 331, $794-814$

Iglesias, C.A., \& Rogers, F.J.: 1991, Astrophys. J. 371, 408 - 417

Iglesias, C.A. \& Rogers, F.J.: 1992, in Astrophysical Opacities, eds. C. Mendoza \& C. Zeippen (Revista Mexicana de Astronomía y Astrofísica) $161-170$

Iglesias, C.A., Rogers, F.J. \& Wilson, B.G.: 1987, Astrophys. J. 322, L45

Kosovichev, A.G., Christensen-Dalsgaard, J., Däppen, W., Dziembowski, W.A., Gough, D.O., \& Thompson, M.J.: 1992, Mon. Not. R. astr. Soc., in the press

Kraeft W.D., Kremp D., Ebeling W. \& Röpke G.: 1986, Quantum Statistics of Charged Particle Systems, Plenum, New York

Krasnikov Yu.G.: 1977, Zh. Eksper. teoret. Fiz. 73, 516 (English translation: Soviet Phys. - JETP 46, 270 - 274; author's name misspelt as "Karsnikov")

Mayer, J.E.: 1950, J. Chem. Phys. 181426 - 1436

Mihalas, D., Däppen W. \& Hummer, D.G.: 1988, Astrophys. J. 331, $815-825$

Reichl, L.E.: 1980, A Modern Course in Statistical Physics, University of Texas Press, Austin

Rogers, F.J.: 1977, Phys. Lett. 61A, $358-360$

Rogers, F.J.: 1981, Phys. Rev. A24, $1531-1543$

Rogers, F.J.: 1986, Astrophys. J. 310, $723-728$

Rouse, C.A.: 1983, Astrophys. J. $272377-379$

Saumon, D. \& Chabrier, G.: 1991, Phys. Rev A44, 5122

Saumon, D. \& Chabrier, G.: 1992, Phys. Rev A, (in press)

Seaton, M.: 1987, J. Phys. B: Atom. Molec. Phys. 20, 6363-6378

Seaton, M.: 1990, J. Phys. B: Atom. Molec. Phys. 23, $3255-3296$

Seaton, M.J.: 1992, in Astrophysical Opacities, eds. C. Mendoza \& C. Zeippen (Revista Mexicana de Astronomía y Astrofísica) 180

Wiese, W.L., Kelleher, D.E. \& Paquette, D.R.: 1972, Phys. Rev. A6 1132 1153 